\title{
Du Moi comme apophatisme: Maurice Barrès, Emil Cioran, Philippe Muray
}

\section{Me as an apophatism: Maurice Barrès, Emil Cioran, Philippe Muray}

\author{
David Paigneau \\ Université de Caen-Normandie \\ david.paigneau@lilo.org
}

\begin{abstract}
In the theological field, the term apophatism refers to a negative approach of defining God by what he is not rather than by what he is. This way of thinking can be applied to the definition of the writer's $M e$ as it appears in the literary works, since the Me, although being a factual obviousness, can only be defined by being opposed to anything it is not. Maurice Barrès, Emil Cioran and Philippe Muray approached, through this oppositional way, the question of the writer's Litterary Me; this comparative study will attempt to confront their respective approaches to the Me and to its inscription in the historical time.
\end{abstract}

\section{Key-words}

Maurice Barrès, Emil Cioran, Philippe Muray, Me, Apophatism.

\begin{abstract}
Résumé
En théologie, l'apophatisme désigne une approche négative consistant à définir Dieu par ce qu'il n'est pas plutôt que par ce qu'il est. Par analogie, cette méthode de pensée peut s'appliquer à la définition du Moi de l'écrivain tel qu'il apparaît dans l'œuvre littéraire, puisque le Moi, quoiqu'étant une évidence factuelle, ne se laisse définir que négativement, par opposition à tout ce qui est autre que lui-même. Maurice Barrès, Emil Cioran et Philippe Muray ont en commun de s'être penchés, à travers cette approche négative, sur la question du Moi littéraire de l'écrivain; cette étude comparatiste tentera de confronter leurs écritures respectives du Moi et de son inscription dans le temps historique.
\end{abstract}

\section{Mots-clés}

Maurice Barrès, Emil Cioran, Philippe Muray, Moi, Apophatisme. 


$$
\begin{array}{r}
\text { "Par une sorte d'ironie de la nature, exister pour } \\
\text { nous c'est se communiquer, et ce que nous } \\
\text { sommes est incommunicable." } \\
\text { (Nicolas Grimaldi, Traité des Solitudes) } \\
\text { "Que moi oui ça alors c'était vrai oui moi c'était } \\
\text { vrai oui et moi je m'appelle comment pas de } \\
\text { réponse MOI JE M'APPELLE COMMENT } \\
\text { hurlements bon que moi en tout cas oui seul oui." } \\
\text { (Samuel Beckett, Comment c'est) }
\end{array}
$$

\section{Introduction}

Il est de ces mots à la polysémie vertigineuse qui ne se laissent aborder qu'au prix d'un effort préalable de "nettoyage de la situation verbale", pour reprendre l'expression de Paul Valéry. Prenez par exemple "temps", "peuple", "amour". Ou "Moi": cas encore compliqué ici par la spécificité du Moi de l'artiste - ou en l'occurrence, de l'écrivain. C'est qu'en effet, écrire le Moi englobe le sens d'écrire sur soi, mais ne saurait s'y limiter. Compte tenu de cette polysémie, il convient de ne pas prétendre à l'exhaustivité et de nous tourner vers une étude de cas et vers une signification bien délimitée.

La signification choisie ici prendra au pied de la lettre le titre du Culte du Moi de Maurice Barrès, en proposant une analogie entre le Moi de l'écrivain et la notion théologique d'apophatisme (du grec apophasis, négation). Également appelée méthode aphairétique (d'après aphairesis, abstraction), son postulat est qu'un être par nature inconnaissable ne peut se rendre accessible à la raison humaine que par ses attributs négatifs. La théologie apophatique consiste ainsi à esquisser une définition de Dieu, non à travers ce qu'il est, mais à travers ce qu'il n'est pas.

Si la tradition exégétique chrétienne fait remonter les origines de la méthode apophatique au Parménide, il semble que Plotin l'ait, le premier, explicitée dans la cinquième Ennéade. La volonté persistante de parler de l'Un tout en avouant une incapacité à le connaître y est présentée comme un paradoxe, que seule une définition négative serait en mesure de dépasser:

Nous pouvons énoncer quelque chose de lui, mais non l'énoncer lui-même par la parole. Nous ne saurions le connaître ni le saisir par la pensée. - Comment donc en parlons-nous puisque nous ne le saisissons pas? - C'est que, s'il échappe à notre connaissance, il ne nous échappe pas complètement. Nous l'embrassons assez pour énoncer quelque chose de lui sans l'énoncer lui-même, pour dire ce qu'il n'est pas, sans dire ce qu'il est ${ }^{1}$; voilà pourquoi nous employons en parlant de lui des termes qui ne sont propres à designer que des choses inférieures (Plotin, 1861: 57).

1 Italique ajouté. 
Dans la tradition chrétienne, c'est chez Saint Thomas d'Aquin que l'apophatisme se trouve posé comme l'un des trois modes d'accès privilégiés à Dieu - sous le nom de "voie négative", aux côtés des voies positive et éminente. La Somme contre les Gentils résume ainsi les enjeux de l'approche apophatique:

La substance divine, en effet, dépasse par son immensité toutes les formes que peut atteindre notre intelligence, et nous ne pouvons ainsi la saisir en connaissant ce qu'elle est. Nous en avons pourtant une certaine connaissance en étudiant ce qu'elle n'est pas ${ }^{2}$. Et nous approchons d'autant plus de cette connaissance que nous pouvons, grâce à notre intelligence, écarter plus de choses de Dieu (D’Aquin, 1993: 118).

La théologie apophatique une fois mise en lumière, il ne semble pas anodin que Pascal, dans ses Pensées, ait fait apparaître le Moi sous la forme d'une évidence indéfinissable, ne pouvant être entrevu qu'à travers tout ce qui, en lui, échappe à toute tentative de définition:

[...] mais celui qui aime quelqu'un à cause de sa beauté, l'aime-t-il? Non: car la petite vérole, qui tuera la beauté sans tuer la personne, fera qu'il ne l'aimera plus. Et si on m'aime pour mon jugement, pour ma mémoire, m'aime-t-on, moi? Non, car je puis perdre ces qualités sans me perdre moi-même. Où est donc ce moi, s'il n'est ni dans le corps, ni dans l'âme $e^{3}$ ? et comment aimer le corps ou l'âme, sinon pour ces qualités, qui ne sont point ce qui fait le moi, puisqu'elles sont périssables? (Pascal, 2011: 323)

En intégrant la nature à la fois tautologique et indicible du Moi à son œuvre apologétique, Pascal ouvre la porte à une approche aphairétique du Moi de l'écrivain. Car la figure de l'auteur au travail représente, si l'on peut dire, un apophatisme au carré, puisque deux identités distinctes, le Moi social et le Moi littéraire, se superposent dans l'œuvre, sans pouvoir être ni séparées, ni confondues. Aussi, le parti pris de cette étude de cas sera de confronter trois auteurs ayant, de façon ponctuelle ou continue, dans des œuvres de fiction, de pensée spéculative ou d'exégèse, choisi la voie négative pour aborder l'étude du Moi. En effet, Maurice Barrès dans quelques-uns de ses romans, Emil Cioran dans ses aphorismes de "penseur privé" et Philippe Muray dans ses travaux critiques, ont en commun de s'être penchés sur le Moi en l'opposant à tout ce qui menace sa perpétuation. Ainsi, le Moi littéraire apparaît sous leurs plumes respectives, tout à la fois comme une réalité à défendre et comme l'arme employée à sa propre défense. Apophatisme enfin, car le Moi ne peut être réellement approché, chez Barrès, Cioran et Muray, qu'une fois défini comme tout ce que n'est pas ce qui n'est pas le Moi.

Chez Maurice Barrès, le Moi est d'abord un Culte, comme l'annonce le titre de sa première trilogie romanesque (Sous l'Eil des Barbares, Un Homme libre, Le Jardin de Bérénice, publiés entre 1888 et 1891). Le jeune littérateur qui se penche sur le sujet est alors

2 Italique ajouté.

3 Italique ajouté. 
un apprenti dandy baudelairien; l'affirmation et le développement du Moi - au prix d'une discipline quotidienne renvoyant explicitement aux Exercices spirituels d'Ignace de Loyola visent avant tout à pallier à la pauvreté des modèles d'identification proposés par une société tombée dans l'apathie depuis la défaite de 1870-71. Dès la préface, Barrès inscrit moins son objet romanesque dans le cadre d'un culte à proprement parler que dans celui d'un repli sur les seules certitudes laissées intactes par le chaos ambiant:

\begin{abstract}
Notre morale, notre religion, notre sentiment des nationalités, sont choses écroulées, constatais-je, auxquelles nous ne pouvons emprunter de règles de vie, et, en attendant que nos maîtres nous aient refait des certitudes, il convient que nous nous en tenions à la seule réalité, au moi ${ }^{4}$. C'est la conclusion du premier chapitre (assez insuffisant, d'ailleurs) de Sous l'œeil des Barbares.

On pourra dire que cette affirmation n'a rien de bien fécond, vu qu'on la trouve partout. À cela, s'il faut répondre, je réponds qu'une idée prend toute son importance et sa signification de l'ordre où nous la plaçons dans l'appareil de notre logique. Et le culte du moi a reçu un caractère prépondérant dans l'exposition de mes idées, en même temps que j'essayais de lui donner une valeur dramatique dans mon œuvre (Barrès, 1986: 18-19).
\end{abstract}

L'observation du Culte du Moi trouvera son contrepoids dans celle de La Colline inspirée (1913). La figure de Léopold Baillard y offre l'exemple d'un Moi tentant de se mettre au service d'un $N_{o u s}{ }^{5}$ mais échouant dans sa tâche, en partie à cause de sa propre infatuation, confirmant ainsi rétrospectivement que le dandysme égocentré mis en scène vingt-cinq ans plus tôt n'était pas ou plus, aux yeux de l'auteur, un idéal en soi mais sa réponse à un certain état social et moral. Ainsi, le Moi apparaît chez Barrès comme une arme à la fois défensive - contre une époque menaçant d'annihiler l'indépendance d'esprit et le sentiment de la grandeur - et offensive - conditionnant la formation d'idées propres et, en définitive, la création littéraire. Toutefois, ce Moi barrésien ne s'oppose au Nous de la collectivité que dans la mesure où le second met en péril la perpétuation du premier; la quête de Barrès s'avère être en réalité celle d'un Nous digne d'être sublimé par des individualités fortes.

L'évolution d'Emil Cioran révèle une trajectoire sensiblement différente: les écrits de la période roumaine, à commencer par l'ouvrage inaugural Sur les Cimes du Désespoir (1934), donnent la parole à un Moi exacerbé qui deviendra, dans les écrits en français, l'objet d'un perpétuel dénigrement, rejoignant les autres sujets récurrents du penseur (la morale, l'Histoire, la philosophie, etc.) dans leur désubstantialisation ironique sous les coups répétés de la conscience de l'absurde. Pour autant, le ton sarcastique ne suffira jamais, chez Cioran, à délégitimer totalement le Moi comme sujet pensant ni comme objet de pensée: c'est au final sous la forme d'une illusion indépassable que le Moi se cristallise au fil des aphorismes, renforçant par-là même la profondeur tragi-comique de l'absurdité universelle. Le Moi qui

4 Italique ajouté.

5 En voulant rendre sa juste place à la colline de Sion. 
se complaît à railler son inconséquence s'enracine et se développe précisément au fur et à mesure qu'il se rengorge de sa lucidité sur lui-même:

La certitude de n'être qu'un accident m'a escorté dans toutes les circonstances, propices ou contraires, et si elle m'a préservé de la tentation de me croire nécessaire, elle ne m'a pas en revanche tout à fait guéri d'une certaine infatuation inhérente à la perte des illusions (Cioran, 2007: 1335).

Je suis requis par la philosophie hindoue, dont le propos essentiel est de surmonter le moi; et tout ce que je fais et tout ce que je pense n'est que moi et disgrâce du moi (Idem: 1276).

Ainsi, la définition du Moi cioranien comme un apophatisme appelle aussitôt une précision: le Moi chez Cioran se caractérise certes par un contraste avec ce à quoi il s'oppose, mais plus encore par sa propre nature de négation incarnée. Sa présence tenace contredit frontalement la pensée de l'auteur tout en étant sa condition d'existence: ce paradoxe insoluble constitue le principal moteur de l'écriture. Outre les Cimes du Désespoir, c'est dans La Tentation d'exister (1956), Histoire et Utopie (1960), La Chute dans le Temps (1964) et De l'Inconvénient d'être né (1973), que le penseur revient le plus longuement sur cette question.

Philippe Muray, en abordant le Moi essentiellement dans des textes consacrés à l'étude d'autres écrivains et artistes que lui-même, l'a ainsi posé comme une problématique structurante d'histoire littéraire plus que comme une obsession personnelle. Mais l'ampleur de son œuvre critique nous autorise très largement à y voir un portrait en creux de l'auteur: dès lors, il apparaît clairement que la puissance d'affirmation du Moi est le plus petit dénominateur commun conditionnant les admirations et les rejets de l'exégète. Les quatre volumes des Exorcismes spirituels (recueils d'articles parus respectivement en 1997, 1998, 2002 et 2005) tissent une pensée du Moi à travers des portraits ciblés s'ouvrant sur des considérations plus générales. Dans le même esprit, l'essai Céline (1981) illustrait déjà cette pensée par l'étude d'un auteur au Moi à la fois tourmenté, hypertrophié, admirable et détestable. Ainsi, de même que chez Barrès, le Moi littéraire constitue à ses yeux la pierre d'achoppement qui sépare irrémédiablement l'individu créateur de la collectivité; mais là où Barrès voyait dans cette dichotomie une contingence qu'il s'agissait de dépasser, Muray la perçoit comme le privilège - amer - de l'écrivain et la source véritable de l'écriture: une perspective plus proche de celle de Cioran, mais dénuée du caractère paradoxal propre à l'existence même du Moi littéraire dans la vision du penseur roumain:

En littérature comme dans tout le reste, le premier mouvement est le mauvais. Ce qui vient à l'esprit d'abord est toujours médiocre, niais, comme parasité ou détrempé par l'horrible pensée des autres, leur vision en commun, leur communauté de stéréotypes. Écrire, c'est chasser l'Autre de la page, méthodiquement, patiemment, le traquer ligne à ligne, d'adjectifs en adverbes, le déloger des métaphores, le mettre en fuite sous l'accumulation d'antithèses astronomiques, le débusquer derrière chacune de ses propres idées, le balayer par des ironies plongeantes et des paradoxes vertigineux. Ce qu'on ap- 
pelle un style, contrairement à ce que tout le monde imagine, n'est que le résultat luxuriant de cette expulsion systématique. Il n'y a pas de grande écriture sans ostracisme, pas de véritable rythme écrit sans discrimination. Rien n'est moins philanthropique ou caritatif que la littérature, qui commence toujours par l'élimination de la spontanéité, c'est-à-dire par l'anéantissement de ce qui nous habite avant tout le reste, cet amas ressassé d'opinions reçues, d'images archi-éculées et de jugements triviaux qui parle à travers chacun d'entre nous. L'Autre, le sacro-saint Autre, c'est l'autre nom de l'anti-littérature. (Muray, 2010: 615).

Le Moi ayant cette particularité de représenter une positivité absolue tout en ne pouvant se définir que négativement; et la création artistique et littéraire, celle de trouver son origine dans une négativité tout en visant à la production d'un contenu positif, la définition négative du Moi littéraire par ce qui le distingue du Nous constituera logiquement le premier moment de cette étude comparatiste. Dans la vision propre à chacun des trois auteurs, cette séparation ontologique apparaît comme indispensable à l'existence de l'écriture, qui tout à la fois découle de l'incompatibilité du Moi et du Nous, la matérialise et finalement, l'aggrave. Toutefois, cette opposition, absolue d'un point de vue théorique, n'exonère pas, en pratique, les auteurs de leur participation à la vie collective, ni de l'inscription de l'œuvre dans le temps historique et dans sa séquence contemporaine. Aussi la seconde étape de la comparaison consistera-t-elle à confronter trois visions de la perception de l'Histoire par le Moi littéraire.

\section{Le Moi et le Nous: exacerbation, fructuosité et dépassement d'un conflit}

La vraie indépendance consiste ainsi dans l'unité et la pénétration réciproque de l'individuel et du général, le général acquérant une existence concrète et s'individualisant, et la subjectivité de l'individuel et du particulier ne trouvant que dans le général une base solide et un contenu véritable pour sa réalité."

(G. W. F. Hegel, L'Idée du Beau)

"L'infinie stupidité des masses me rend indulgent pour les individualités, si odieuses qu'elles puissent être.'

(Lettre de Gustave Flaubert à Georges Sand)

Quand ces hommes que les Romains appelaient ennemis du genre humain pénétrèrent dans les temples et fracassèrent les têtes de bois des idoles païennes, ils virent s'en échapper des nichées de souris arrachées à l'ombre confortable et sacrée où elles se reproduisaient depuis des siècles (Muray, 2001: 23).

Ouvrant par cette phrase son essai sur Céline, Philippe Muray lui donne valeur de parabole: les nichées de souris y représentent la communauté et les idoles, les mensonges 
admis au nom de la cohésion collective. Quant aux barbares fracassant les têtes des idoles, ils sont le visage prêté par l'essayiste à la figure de l'écrivain, dont le destin est de s'écarter de la collectivité pour, n'ayant plus rien à gagner à la perpétuation de son unité, ébranler méthodiquement les illusions rassurantes sur lesquelles elle repose. Muray, en posant dès l'ouverture du livre l'écrivain et la communauté comme deux ennemis irréductibles, établit une grille de lecture applicable à l'ensemble de ses textes critiques, tout en croisant la route d'une large partie des œuvres barrésienne et cioranienne: celle de la définition du Moi littéraire comme une négativité incarnée, entrant avec le Nous dans un rapport d'hostilité affichée et d'interdépendance souterraine. C'est donc entre deux pôles, deux frontières théoriques, le dans absolu et le contre absolu, qu'oscille la présence du Moi au sein du Nous.

Le parcours de Maurice Barrès témoigne de ce point de vue d'une évolution, constante à défaut d'être linéaire, transformant petit à petit le dandy jouisseur et désabusé du Culte $d u$ Moi en un "professeur d'énergie" mettant sa plume au service du moral de guerre dans ses chroniques pour l'Écho de Paris en 1914-1918; en d'autres termes, d'un passage de la volonté de séparation à la quête d'une fusion entre le Moi et le Nous. Mais les signes avant-coureurs de cette évolution sont décelables dès la trilogie romanesque de 1888-1891.

Sous l'Eil des Barbares met en scène Philippe, jeune apprenti intellectuel s'initiant à la vie parisienne. À la fois héritier de Rastignac pour l'ambition et de Rubempré pour la perte sèche de ses illusions, double du romancier pour la ligne de conduite vers laquelle son désenchantement l'engage, le personnage découvre dans son Moi un objet de culte paradoxal, ne se révélant pas par des manifestations de toute-puissance, mais au contraire par sa vulnérabilité face à tout ce qui contredit ses postulats et conditions d'existence. Ces menaces permanentes prennent le nom de "Barbares"; plus précisément, elles occupent l'espace qui sépare les espérances du jeune et naïf dandy et la réalité des êtres qui croisent sa route. Le regard que Philippe porte sur ces espoirs devenus hostiles, puis la conclusion que seule une séparation au moyen d'une singularisation forcenée lui permettra de préserver son Moi des dangers de l'indifférenciation, trouvent plusieurs échos dans les écrits de Cioran et Muray.

Dans le roman, ces menaces s'incarnent essentiellement sous deux formes, celle des femmes et celle des maîtres à penser dévoyés. Les premières jouent dans la trilogie un rôle dont l'évolution répond à celle de Philippe à chaque étape de son édification. De manière très nette, la présence féminine apparaît comme un obstacle à proportion que le personnage considère l'affirmation de son Moi comme une fin en soi. Ainsi, c'est sa maîtresse, en louant sa beauté, qui fait, dans un premier temps, prendre conscience à Philippe de la fragile essence de son Moi et de la nécessité de lui donner une définition substantielle:

$\mathrm{Ah}$ ! combien je le prévoyais! vous daignez goûter quelques formes où j'habite, et jamais vous n'atteindrez à m'aimer moi-même, car votre caprice peut-être ne soupçonne même pas sous mes apparences mon âme. Ah! mon incertaine beauté qui n'est qu'un reflet de votre jeunesse! ma parole, ce masque que ne peut rejeter ma pensée! mes in- 
certitudes, où trébuche mon élan! tous ces sentiers que je piétine! tout ce vestiaire, c'est donc vers cela que tu soupirais, pauvre âme? (Barrès, 1986: 54)

Dans Un Homme libre, le personnage intègre une théorisation de sa relation aux femmes au sein de la discipline par laquelle son Moi doit s'exercer et s'affirmer. Les préceptes qu'il en tire appliquent en somme le "tout désirer, tout mépriser" barrésien à ses aventures sentimentales:

\begin{abstract}
Jouissons et dansons, mais voyons clair. Il faut traiter toutes choses au monde comme les gens d'esprit traitent les jeunes filles. Les jeunes filles, au moins en désir, se sont prêtées à tous les imbéciles, et lors même qu'elles sont vierges de désir, croyez-vous qu'il n'existe pas un imbécile qui puisse leur plaire! Il faut faire un assez petit cas des jeunes filles, mais nous émouvoir à les regarder, et nous admirer de ressentir pour de si maigres choses un sentiment aussi agréable (164).
\end{abstract}

Le Jardin de Bérénice voit ces représentations mises en doute au fur et à mesure que Philippe lie le destin de son Moi à celui d'un Nous par le biais de l'action politique. Dès lors, Bérénice, aimée de Philippe mais future épouse de son rival électoral Charles Martin, personnifie à ses yeux les raisons qui l'attirent spontanément vers la foule: "Par cette tension que lui donnent son chagrin et son regret sans réalité, elle atteint un objet qu'elle n'a pas visé. Ah! C'est bien elle, la chère petite fille, qui m'a aidé à comprendre la méthode créatrice des masses, de l'homme spontané!" (319).

L'identification de la féminité au Nous est commune aux trois auteurs; c'est parce qu'elle accompagne, chez Barrès, un parcours qui va de l'affirmation au dépassement du Moi que cette identification perd progressivement une partie de son caractère péjoratif. De sorte qu'une différence fondamentale entre le romancier et Muray - le fait que, chez ce dernier, le Moi de l'écrivain demeure une fin en soi et ne tende pas à s'élargir au Nous - se trouve reproduite dans le caractère non-évolutif des représentations féminines tout au long des Exorcismes spirituels: la place accordée par le Moi au Nous semble ainsi conditionner, aux yeux des deux auteurs, le statut de la femme dans l'œuvre. Chez Muray toutefois, ce statut sert avant tout à introduire une question plus vaste: celle de la différenciation sexuelle, moteur indispensable de la permanence du conflit, lui-même à la source de la continuité historique et, en définitive, de la force vitale. Ainsi, le positionnement de l'artiste par rapport à la guerre des sexes surdétermine la puissance d'affirmation de son Moi. Un exemple particulièrement révélateur se trouve dans les Exorcismes spirituels I, lorsque Muray entreprend de comparer les entrées "Célibataire" et "Génie" dans le Grand Dictionnaire de 1876: les deux termes y sont définis quasiment à l'identique, comme des névroses et des pathologies; "l'homme entièrement possesseur de ses moyens" est "l'honnête bourgeois", par opposition au "génie" qui "se croit appelé à d'autres destinées que les êtres de son espèce" et "[viole] les lois primordiales" de la vie ordinaire qu'il "dédaigne»: "enfin, pour tout dire en un mot, 
il est célibataire!" (Muray, 2010: 683-684). L'auteur en tire une conclusion renvoyant en droite ligne au Culte du Moi: la femme étant le visage de la collectivité, elle-même régie par la poursuite de ses intérêts propres et incompatibles avec ceux de l'individu, sa proximité ne peut représenter pour le Moi résolument égoïste et singulier de l'artiste qu'une menace existentielle. Muray illustre sa thèse par une anecdote mettant en scène Edgar Degas, lequel justifiait son choix du célibat par la crainte de voir une femme entrer dans son atelier et complimenter son travail (683).

La position de Cioran sur le rapport entre le Moi et les femmes ne peut se déduire que d'une absence, car le penseur roumain ne s'est que très peu penché sur cette question. Ce silence peut néanmoins constituer un début de réponse, si l'on observe que les rares textes consacrés à la féminité se trouvent dans Les Cimes du Désespoir, soit dans un livre antérieur au dénigrement systématique du Moi comme du Nous. "L'essence de la grâce" établit un postulat relativement proche des positions de Barrès et Muray:

\begin{abstract}
La femme ne risque rien sur le plan spirituel, car chez elle l'antinomie de la vie et de l'esprit a une intensité moindre que chez l'homme. Le sentiment grâcieux de l'existence ne mène point aux révélations métaphysiques, à la perspective des derniers instants ni à la vision des réalités essentielles, qui vous font vivre comme si vous ne viviez plus. Les femmes déconcertent: plus on pense à elles, moins on les comprend. Processus analogue à celui qui vous réduit au silence à mesure que vous réfléchissez sur l'essence ultime du monde. Mais tandis que vous restez, en ce cas, abasourdi devant un infini indéchiffrable, le vide de la femme vous apparaît comme un mystère. La femme a pour mission de permettre à l'homme d'échapper à la pression torturante de l'esprit; elle peut être un salut. À défaut d'avoir sauvé le monde, la grâce aura au moins sauvé les femmes (Cioran, 2007: 59).
\end{abstract}

D'après le Cioran de la période roumaine, la grâce constitue donc la principale qualité par laquelle la femme se distingue de l'homme et lui offre la possibilité d'échapper à ses tourments spirituels - à "la pression torturante de son esprit". Cependant, ce "sentiment grâcieux" recouvre un "vide" qui "ne mène point aux révélations métaphysiques" ni à "la vision des réalités essentielles, qui vous font vivre comme si vous ne viviez plus". Or, ces vérités essentielles sont précisément la condition d'existence du Moi, qui ne se définit chez Cioran que par le tourment causé par un regard lucide et acerbe porté sur la réalité de l'existence. Au final, cette lucidité se retournera, dans les écrits ultérieurs, contre la substance même du Moi. Les différentes approches de la question féminine chez les trois auteurs semblent donc cacher un paradigme partagé, assimilant la féminité au Nous face auquel doit se positionner le Moi. La nature plus évolutive de la vision barrésienne de la femme par rapport à la vision murayienne tient donc à l'évolution parallèle de la nature et de la fonction du Moi sous sa plume. Chez Cioran, le thème de la féminité subit au fil du temps le même processus de désubstantialisation que les questions conjointes du Moi et du Nous, confirmant donc - mais négativement, en somme par contraposée - l'existence d'un parallèle. 
Dans Le Culte du Moi, la question féminine rejoint celle des maîtres à penser dévoyés, puisque c'est la maîtresse de Philippe qui lui fait rencontrer M. X., un vieillard et philosophe donnant à son potentiel disciple une série de conseils devant l'élever jusqu'aux hautes sphères de la vie culturo-mondaine. Rappelant Vautrin comme Philippe rappelle Rastignac ou Rubempré, son mentor autoproclamé l'enjoint à ne jamais perdre de vue ses ambitions, à faire le bilan de ses forces et de ses faiblesses et à établir un agenda devant le conduire à la réussite, sans se laisser circonvenir par les séductions du monde extérieur: "Mais gardez de laisser cet agenda sur l'oreiller d'une amie qui s'étonne et admire, ou dans le verre d'un camarade qui s'écrie: 'Moi aussi..."” (Barrès, 1986: 87). Le Moi que M. X. se propose d'édifier n'est donc pas à proprement parler le Moi littéraire, mais le Moi social en quête de reconnaissance littéraire:

Que désormais chacun découvre, et à votre attitude seule, combien vous êtes né pour ce but que secrètement vous vous fixez. Vos fréquentations, la coupe de vos vêtements contribueront à créer l'opinion. Soignez vos manies, vos partis pris et vos ridicules; c'est l'appareil où se trahit un spécialiste. De là sera déduit votre caractère (87).

La réaction de Philippe, chassant le vieillard après l'avoir battu à coups de canne (90), révèle que $\mathrm{M}$. X. a été perçu par lui comme un "Barbare": autrement dit, non comme un être extérieur ou encore moins inférieur à ses propres préoccupations - l'intelligence conceptuelle et la connaissance des hommes étant de fait plus développées chez le philosophe chevronné que chez l'apprenti dandy -, mais comme une incarnation du Nous indifférent à l'authenticité du Moi de Philippe. Une distinction que Barrès détaille dès la préface du roman:

Si Philippe se plaint de vivre 'sous l'œil des barbares', ce n'est pas qu'il se sente opprimé par des hommes sans culture ou par des négociants; son chagrin c'est de vivre parmi des êtres qui de la vie possèdent un rêve opposé à celui qu'il s'en compose. Fussent-ils par ailleurs de fins lettrés, ils sont pour lui des étrangers et des adversaires [...]. Ainsi, quand on les oppose, prennent leur pleine intelligence ces deux termes Barbares et Moi. Notre moi, c'est la manière dont notre organisme réagit aux excitations du milieu et sous la contradiction des Barbares (20).

L'attitude décrite ici relève bien du domaine de l'apophatisme, puisque c'est dans la confrontation avec son opposé que peut s'esquisser une définition du Moi. La parabole de Muray y apparaît en creux, mais à front renversé: chez le Barrès de 1890, l'idole incarnerait l'idéal du Moi poursuivi par l'écriture littéraire, les Barbares seraient, plus explicitement, les injonctions de la communauté - fût-ce la communauté culturelle ou intellectuelle - et les souris en déroute matérialiseraient le risque de morcellement et de fuite du Moi sous les assauts répétés du Nous. Confrontation que l'on peut également identifier chez Cioran, à cette nuance près que le Moi, après s'être construit dans l'opposition au Nous, se voit finalement emporté par le même processus de négation. Ainsi, revenant sur les postulats des Cimes du 
Désespoir dans la lettre à Constantin Stoïca qui ouvre Histoire et Utopie, le penseur persiste dans l'idée que "Vivre véritablement, c'est refuser les autres" (Cioran, 2007: 981). Mais ce refus dissimule mal une dépendance que La Tentation d'exister, un an plus tôt, détaillait en faisant de la matérialisation de l'adversaire sous la forme de l'Autre, la condition d'existence et la source vitale du Moi:

Si j'oppose l'Unité, seule véridique, à la multiplicité, nécessairement mensongère, si, en d'autres termes, j'assimile l'autre à un fantôme, ma révolte se vide de sens, elle qui, pour exister, doit partir de l'irréductibilité des individus, de leur condition de monades, d'essences circonscrites. Tout acte institue et réhabilite la pluralité, et, conférant à la personne réalité et autonomie, reconnaît implicitement la dégradation, le morcellement de l'absolu (828).

Deux aphorismes proches, dans De l'Inconvénient d'être né, illustrent parfaitement ce paradoxe: définissant tout d'abord "Autrui" comme "une invention de dialecticien" (1291), le penseur résume ensuite la «connaissance de soi" à "une contradiction dans les termes" (1292). Dans la vision cioranienne, l'affirmation du Moi par opposition au Nous possède sans doute la même efficacité théorique que chez Barrès et Muray; mais cette efficacité n'élève finalement pas le Moi au-dessus de sa propre nature illusoire: "allié de circonstance" dans la négation du Nous, le Moi, pensé en dehors de cette opposition, perd du même coup sa substance propre.

'Méditez seulement une heure sur l'inexistence du moi et vous vous sentirez un autre homme', disait un jour à un visiteur occidental un bonze de la secte japonaise Kousha. Sans avoir couru les couvents bouddhiques, combien de fois ne me suis-je pas arrêté sur l'irréalité du monde, donc du moi? Je n'en suis pas devenu un autre homme, non, mais il m'en est resté effectivement ce sentiment que mon moi n'est réel en aucune façon, et qu'en le perdant je n'ai rien perdu, sauf quelque chose, sauf tout (1282).

Le "Nous" est ainsi nécessaire à la définition du "Moi", mais cette interdépendance n'aboutit chez Cioran qu'à occulter une inconséquence partagée. Dans cette idiosyncrasie, les véritables maîtres à penser sont les sages orientaux dont le paradigme repose précisément sur l'abandon du Moi en tant qu'illusion. C'est donc au nom de ce qui est présenté comme une vérité factuelle, et non de la conformité aux règles imposées par une communauté, qu'ils ébranlent l'édifice sur lequel repose la persévérance dans l'être du Moi littéraire. En conséquence, tout écrivain posant son Moi comme une évidence et un relais fiable entre lui et le monde, dégénère immanquablement dans le narcissisme verbeux et stérile et se voit disqualifié en tant que potentiel "maître à penser":

Le littérateur? Un indiscret qui dévalorise ses misères, les divulgue, les ressasse: 1'impudeur - parade d'arrière-pensées - est sa règle: il s'offre. Toute forme de talent va de pair avec un certain sans-gêne. N'est distingué que le stérile, celui qui s'efface avec son 
secret, parce qu'il dédaigne de l'étaler; les sentiments exprimés sont une souffrance pour l'ironie, une gifle à l'humour (880).

L'on décèle aisément chez Philippe Muray un même dévoiement des hauts pontes de la vie littéraire; par ailleurs, ce discrédit trouve, de même que chez Cioran, sa source dans un narcissisme réduit à tourner à vide. Un rapprochement qui saute aux yeux si l'on confronte ces deux textes extraits respectivement de La Tentation d'exister et des Exorcismes spirituels III:

Du temps que l'artiste mobilisait toutes ses tares pour produire une œuvre qui le cachait, l'idée de livrer sa vie au public ne devait même pas l'effleurer. On n'imagine pas Dante ni Shakespeare notant les menus incidents de leur existence pour les porter à la connaissance des autres. Peut-être même tendaient-ils à donner une fausse image de ce qu'ils étaient. Ils avaient cette pudeur de la force que le déficient moderne n'a plus [...]. Nul besoin, chez eux, de s'inventer une vie: ils existaient; ce qui n'arrive point au civilisé. Décidément, nous ne saurons jamais pourquoi nos ancêtres ne se sont pas barricadés dans leurs cavernes (Cioran, 2007: 902).

Il faut avoir beaucoup à dissimuler pour avoir quelque chose d'intéressant à montrer. Et si très peu de gens sont intéressants, aujourd'hui, quand ils se montrent par leurs écrits, c'est que l'on sent aussitôt qu'ils ont également très peu à cacher. (Muray, 2010: 1314).

L'antagonisme entre l'affirmation du Moi par l'écriture et le narcissisme pur et simple tient dans la possibilité de distinguer le Moi social du Moi littéraire: le nombrilisme repose sur leur indistinction, le tempérament littéraire sur leur distanciation - de même, c'est pour n'avoir pas clairement posé cette dichotomie que M. X. s'attire les foudres de Philippe. Mais la principale différence entre les perspectives de Cioran et Muray vient de ce qu'aux yeux du second, le Moi littéraire, faisant déjà figure de "malédiction" aux yeux du Moi social, échappe partiellement à la condamnation prononcée par la conscience de l'absurde. De plus, c'est parce que le Moi social de l'écrivain chérit et protège son Moi littéraire tout en le maudissant, qu'il apparaît aux yeux de la collectivité comme une absurdité incarnée, entérinant ainsi la séparation entre le Moi et le Nous. Un paradoxe qui s'éclaire dans deux passages des Exorcismes spirituels I: d'abord dans le constat que l'amour de la littérature n'est pas, loin s'en faut, le sentiment le mieux partagé chez les écrivains de rang supérieur; ensuite dans le commentaire de deux aphorismes de Nietzsche postulant que la recherche de la santé représente l'antithèse du génie:

Un écrivain n'est d'ailleurs pas nécessairement quelqu'un qui 'aime' la littérature, il peut même se payer le luxe de laisser cette passion flatteuse à son entourage, tout cela n'a aucune importance, il s'agit d'autre chose, mais le malentendu sera éternel, inutile de se faire des illusions (Muray, 2010: 570).

Que la santé et la moralité soient incompatibles avec le génie n'a rien de si surprenant: le sens commun, à l'opposé, n'assimile-t-il pas le génie à un phénomène morbide ou à un dérapage pathologique, quand ce n'est pas à une forme insaisissable de délin- 
quance? [...] Personne ne veut le génie parce que personne n'a intérêt à souffrir. Au commencement, donc, était le bien-être absolu, et tout doit être mis en œuvre pour le restaurer comme un paradis perdu organique dont le retour dans les plus brefs délais fait partie de nos droits acquis (675).

Ici, le véritable point de convergence entre Barrès, Cioran et Muray tient dans une opposition entre le quantitatif et le qualitatif, sous la forme d'une confrontation entre les "maîtres à penser" du Nous et ceux du Moi. Chez Barrès, l'antagonisme apparaît de façon explicite dans le cinquième chapitre d'Un Homme libre, "Les intercesseurs" (Barrès, 1986: 166-180). Penseurs et écrivains reconnus par Philippe comme les intermédiaires privilégiés entre lui et son Moi, ces intercesseurs doivent lui permettre de dépasser "l'Intelligence souffrante" pour atteindre à "l'Intelligence triomphante" (166-167). Benjamin Constant et SainteBeuve $^{6}$ sont ainsi les objets d'un culte pour ainsi dire "transitoire", étape indispensable dans le perfectionnement du culte du Moi:

Chers esprits excessifs, les plus merveilleux intercesseurs que nous puissions trouver entre nous et notre confus idéal, pourquoi confesserais-je le culte que je vous ai! Vous n'existez qu'en moi. Quel rapport entre vos âmes telles que je les possède et telles que les dépeignent vos meilleurs amis! Il n'est de succès au monde que pour celui qui offre un point de contact à toute une série d'esprits. Mais cette conformité que vos vulgaires admirateurs proclament me répugne profondément. Vous n'atteignez à me satisfaire qu'aux instants où vous dédaignez de donner aucune image de vous-mêmes aux autres, et quand vous touchez enfin ce but suprême du haut dilettantisme, entrevu par l'un des plus énervés d'entre vous: 'Avant tout, être un grand homme et un saint pour soimême...' Pour soi-même!... dernier mot de la vraie sincérité, formule ennoblie de la haute culture du moi qu'à Jersey nous nous proposions. (179).

Les "vulgaires admirateurs" des "intercesseurs" forment ainsi un autre visage des "Barbares", poursuivant Philippe jusque dans ses admirations intéressées. Un contraste déjà à l'œuvre dans Sous l'Eil des Barbares, lorsque Philippe détaille les modèles littéraires des “jeunes esprits curieux»: Heine, Taine, le Flaubert de La Tentation de Saint-Antoine et le Baudelaire des Fleurs du Mal (39). Point commun entre tous ces auteurs, se rapprocher de leurs œuvres implique déjà de réaliser un "pas de côté» par rapport à une époque qui se refuse à les intégrer: "Là est le grand secret de l'éducation d'un jeune homme; il s'attache aux auteurs qu'on prétendait ne lui faire connaître que pour les accabler à ses yeux" (40). Vouer un culte au Moi nécessite donc d'opposer ces intercesseurs à la cohorte des littérateurs qui ont formé l'esprit des M. X. et des Charles Martin, dont l'indépendance d'esprit se résume en définitive à "répéter des formules prises au cabinet de lecture" (18).

Chez Cioran également, lecture, pensée et écriture répondent à des enjeux existentiels qui dépassent de très loin la constitution d'un mince vernis de culture raffinée. Cet aphorisme de La Chute dans le Temps résume la vision d'un goût pour l'art et la littérature réduit à la

6 Plus précisément le Sainte-Beuve de 1835, autrement dit le poète par opposition au critique (Barrès, 1986: 174). 
Anales de Filología Francesa, n. ${ }^{\circ}$ 27, 2019

Du Moi comme apophatisme: Maurice Barrès, Emil Cioran, Philippe Muray

tentation d'une recherche d'approbation de soi par l'autre, censé pallier aux incertitudes du Moi:

\begin{abstract}
Si chacun de nous avouait son désir le plus secret, celui qui inspire tous ces projets et tous ses actes, il dirait: 'Je veux être loué.' Nul ne s'y résoudra, car il est moins déshonorant de commettre une abomination que de proclamer une faiblesse aussi pitoyable et aussi humiliante, surgie d'un sentiment de solitude et d'insécurité dont souffrent, avec une égale intensité, les rejetés et les chanceux. Personne n'est sûr de ce qu'il est, ni de ce qu'il fait. Si imbus que nous soyons de nos mérites, nous sommes rongés par l'inquiétude et ne demandons, pour la surmonter, qu'à être trompés, qu'à recevoir de l'approbation de n'importe où et de n'importe qui (Cioran, 2007: 1113).
\end{abstract}

Les véritables enjeux de la plongée dans la pensée étaient en réalité posés dès les Cimes du Désespoir: “À celui qui pense pour le plaisir de penser s'oppose celui qui pense sous l'effet d'un déséquilibre vital” (32). Or, si les écrits ultérieurs de Cioran contredisent sur de nombreux points les positions affirmées en 1934, cette ligne de fracture fondamentale parcourt en revanche toute l'œuvre. Ainsi, encore dans De l'Inconvénient d'être né, l'écriture apparaît comme l'objectivation d'une quête du Moi: “Aller jusqu'aux extrémités de son art et, plus encore, de son être, telle est la loi de quiconque s'estime tant soit peu élu" (1302). Une telle ambition est inaccessible à la figure de l'artiste et de l'écrivain contemporains, résumés aux yeux du penseur par leur dimension quantitative. L'aphorisme suivant peut avantageusement être confronté à un texte extrait des Exorcismes spirituels I pour mettre en évidence la proximité entre les visions cioranienne et murayienne: “À mesure que l'art s'enfonce dans l'impasse, les artistes se multiplient. Cette anomalie cesse d'en être une, si l'on songe que l'art, en voie d'épuisement, est devenu à la fois impossible et facile" (1302).

Quand le monde s'artistise, c'est l'art qui perd son autre, son 'ombre', son contraire; pas seulement ses ennemis, mais le monde lui-même, le monde étranger, tout ce qui n'était pas lui. L'artistisation de la société entraîne la destitution de l'art par effacement de toute distinction entre art et non-art. L'art n'a plus de sexe opposé. (Muray, 2010: 770).

Philippe Muray, en mêlant intimement son activité de critique littéraire à celle de chroniqueur au vitriol des mœurs contemporaines, est, des trois auteurs, celui qui a le plus longuement développé ce sujet. L'opposition frontale entre les dimensions quantitative et qualitative des "maîtres à penser" est l'un des topoï qui traversent ses écrits de la façon la plus récurrente. Qu'il s'agisse des “Mutins de Panurge" (771) ou des “artistocrates” (1555), les néologismes affleurent sous sa plume pour désigner les édificateurs du Nous au détriment du Moi. À cet égard, le texte "Panique dans la Culture (apologue sur une idée de Dostoïevski)" (765-768) est particulièrement éclairant. S'inspirant du récit "Le Grand Inquisiteur" des Frères Karamazov, dans lequel le Christ, revenu sur Terre sous l'inquisition espagnole, 
est emprisonné par le cardinal comme élément subversif, Muray imagine une irruption des artistes, écrivains et philosophes du passé pendant une manifestation culturelle contemporaine (Salon du Livre, Fête de la Musique ou autre): les importuns se voient chassés par le service de sécurité car incompatibles avec la figure de l'artiste conçu comme positivité et adhésion à la société:

Nous, la police suave et vaselinée de la pensée fondée sur les meilleures intentions, l'amour de l'avenir, du progrès, de la santé, du pouvoir de la femme, du rapprochement entre les peuples et des droits de l'enfant, nous savons que vous avez toujours représenté la désapprobation rampante, l'opposition radicale ou sournoise à ces valeurs de rayonnement [...]. Nous connaissons le NON qui vous résume, et sur lequel nous construisons notre temple du OUI perpétuel. Tout ce qui était rupture chez vous, nous l'avons retraduit en communion. Tout ce qui était solitude déclenche nos fièvres collectives. Tout ce qui était dysharmonie, nous en avons fait nos cantiques. Maintenant, nous pouvons adorer et faire adorer jusqu'à votre inquiétude, votre ironie, vos blasphèmes, puisqu'ils ne sont plus que les signes désolants d'un monde révolu. (768).

Dans les Exorcismes spirituels III, l'article "Malraux versus Culture" oppose l'idée malrucienne de la Culture - un anti-destin à l'adresse du Moi - à son acception contemporaine sous la forme de la bonne conscience collective:

On n'entend plus les Voix du Silence. La voix de harpie de la Culture les a remplacées. Mais nul ne peut tenir Malraux pour responsable de la métamorphose funeste de ce qu'il appelait, lui, culture, en instrument de contrôle et de domestication de cette modernité à laquelle rien ni personne, désormais, ne doit plus échapper [...]. La Culture est l'autre nom de la fête, qui est le cœur disciplinaire de la société qui commence et l'organe par laquelle s'exprime le nouveau Parti de l'Ordre [...]. L'art, qu'il soit littéraire ou plastique, n'exprimait jamais rien d'autre à ses yeux que l'idée que la partie n'est pas jouée, qu'il n'y a pas de lois, que rien ne sera jamais complètement analysé ni bouclé, qu'aucune solution jamais n'en terminera avec le moindre problème, qu'aucune réponse ne comblera jamais le désir insatiable de questions (si possible insolubles). Et il est probable qu'il rêvait de voir offrir à tous cet anti-destin sous le nom de culture. Et qu'il n'aurait jamais imaginé la transformation de cette dernière en programme de soumission des populations à l'avenir qu'on a choisi pour elles. (1157-1158).

De ce point de vue, le cas de Maurice Barrès diffère en ce que, dès le Culte $d u$ Moi, celui-ci aspire bel et bien au titre de "maître à penser" - ce qu'il deviendra effectivement pour plusieurs générations d'écrivains. Volonté qui apparaît nettement dans la "Dédicace" d'Un Homme libre, adressée aux collégiens tentés par le suicide et à qui le roman est supposé fournir une discipline à même de leur rendre la vie plus supportable:

Chercher continuellement la paix et le bonheur, avec la conviction qu'on ne les trouvera jamais, c'est toute la solution que je propose. Il faut mettre sa félicité dans les expériences qu'on institue, et non dans les résultats qu'elles semblent promettre. Amusons-nous aux moyens, sans souci du but. Nous échapperons ainsi au malaise habituel 
des enfants honorables, qui est dans la disproportion entre l'objet qu'ils rêvaient et celui qu'ils atteignent (Barrès, 1986: 127-128).

François Proulx rappelle à ce sujet que l'augmentation du taux de suicide chez les jeunes hommes férus de poésie romantique et de philosophie nihiliste, était un phénomène réel et l'objet de débats houleux dans les années 1885-1895; la présence de la dédicace n'a donc rien de fortuit: 'D'après cette préface, la trilogie du Culte du moi ne se contente pas de représenter le jeune homme de l'époque à titre de renseignement; elle s'adresse à lui à titre d'enseignement, dans l'espoir de corriger le mal qui le ronge» (Proulx, 2011: 28). L'objectif de Barrès vis-à-vis des jeunes lecteurs serait ainsi de constituer "un espace imaginaire supervisé qui permet aux lecteurs de jouir des attraits du nihilisme littéraire tout en les menant à le délaisser" (29). Cette volonté de mettre son Moi au service de la communauté dont il avait dans un premier temps fallu se détacher, se retrouve également dans l'évolution de Philippe. Les derniers chapitres d'Un Homme libre le voient prêt à se mêler aux hommes, d'abord sans rien perdre de son égoïsme constitutif:

J'ai renoncé à la solitude; je me suis décidé à bâtir au milieu du siècle, parce qu'il y a un certain nombre d'appétits qui ne peuvent se satisfaire que dans la vie active. Dans la solitude, ils m'embarrassent comme des soudards sans emploi. La partie basse de mon être, mécontente de son inaction, troublait parfois le meilleur de moi-même. Parmi les hommes je lui ai trouvé des joujoux, afin qu'elle me laisse la paix. (Barrès, 1986: 248).

C'est dans Le Jardin de Bérénice que la trajectoire du Moi au Nous s'achève: candidat aux élections législatives à Arles, Philippe se trouve opposé à Charles Martin; ce dernier, modèle de logique formelle et de rationalité, ne possède aucune des vertus du Moi - son savoir purement impersonnel n'ayant permis aucun développement de son tempérament - et, par conséquent, se montre incapable de comprendre l'âme profonde du segment de population qu'il prétend administrer. Aussi Philippe doit-il expliquer, à son adversaire lui reprochant de faire campagne en se mêlant à un peuple qu'il devrait mépriser comme inférieur à lui-même, que ce contact lui a permis d'élever son Moi au-delà de ses propres qualités en lui révélant la substance humaine, l'énergie créatrice et les ressorts de l'inconscient collectif (314-316). Le développement méthodique du Moi est donc encore considéré par Barrès comme une étape indispensable dans la constitution d'un personnage de "maître à penser" authentique au service de la communauté. Mais La Colline inspirée ira plus loin en voyant dans la présence trop envahissante du Moi de Léopold Baillard, l'une des raisons de son échec à fédérer la communauté lorraine autour de ses idéaux.

La raison pour laquelle les écritures respectives du Moi de Barrès, Cioran et Muray peuvent être réunies sous le terme d'apophatisme, est donc que dans les trois œuvres, cet objet de pensée récurrent qui est également le sujet pensant, ne se laisse constamment approcher que par ce qui l'oppose à ses contraires. Le "Nous"est le plus petit dénominateur commun 
entre lesdits contraires; ce n'est que sur les conclusions que doit tirer le Moi littéraire de cette confrontation avec le Nous que les trois auteurs se séparent relativement: antagonisme à dépasser après l'avoir “crevé" - à la manière d'un abcès - chez Barrès; couple d'entités illusoires, ne pouvant se fabriquer une réalité de circonstance que dans leur confrontation, chez Cioran; dichotomie théoriquement absolue, surdéterminant la substance profonde de l'œuvre, chez Muray. Mais quel que puisse être le désir de sédition que le Moi littéraire exprime à l'égard du Nous, demeure un espace incompressible au sein duquel ces deux réalités doivent nécessairement coexister, puisque l'existence même de l'œuvre inscrit son auteur dans le temps historique - dans une continuité globale tout autant que dans une séquence donnée. Par ailleurs, la présence du Moi de l'écrivain dans l'Histoire est l'une des thématiques récurrentes communes de Barrès, Cioran et Muray. Après avoir tenté de définir l'écriture du Moi propre à chacun des trois auteurs, nous nous pencherons à présent sur la manière dont leurs œuvres respectives développent une pensée de l'Histoire surdéterminée par les caractéristiques du Moi littéraire.

\title{
3. De l'histoire du Moi au Moi dans l'Histoire
}

\author{
"À défaut de la grande histoire que nous ne \\ savons plus faire, nous ramassons les miettes de \\ la petite avec un tel zèle que notre considération \\ en est venue à ouvrir ses grands yeux devant un \\ collectionneur de timbres-poste." \\ (Nadar, Quand j'étais photographe) \\ "Et, petit à petit, vous voilà devenus \\ L'Arc de Triomphe en moins, des soldats incon- \\ nus." \\ (Georges Brassens, Les deux Oncles)
}

À considérer l'évolution du rapport de Maurice Barrès, Emil Cioran et Philippe Muray à l'Histoire via leurs parcours biographiques respectifs, déjà des points de convergence et de divergence apparaissent, qui se trouvent tantôt accentués, tantôt transcendés lorsque la comparaison mêle les vies et les œuvres.

En quelques mots: Barrès et Muray, à un siècle de distance, ont tous deux identifié leur époque comme une sortie du temps historique; mais le premier, "amoureux déçu” de l'histoire de France, voulut insuffler à son écriture l'énergie vitale nécessaire à un retour du Nous français dans l'histoire contemporaine. Le second, après avoir théorisé la fin de l'Histoire au tournant des $\mathrm{XX}^{\mathrm{e}}$ et $\mathrm{XXI}^{\mathrm{e}}$ siècles ${ }^{7}$, l'a traitée comme un fait accompli et irréversible, objecti-

7 Dans Après l'Histoire I \& II, parus respectivement en 1999 et 2000. Par ailleurs, dans plusieurs articles et entretiens, Muray a souligné la différence entre sa définition de la "Fin de l'Histoire» et celle donnée par Francis Fukuyama en 1989 puis 1992. Voir Muray, 2010: 1217. 
vable par la littérature. Muray a également en commun avec Cioran d'avoir, dans sa jeunesse, pris part à un mouvement prétendant écrire un nouveau chapitre historique (la Garde de Fer pour Cioran, l'agitation politique et intellectuelle de l'après-1968 pour Muray) avant de se retirer de toute aventure collective. Mais le penseur roumain, une fois revenu de ses premiers élans, développa un scepticisme radical englobant à la fois le Moi et l'Histoire, là où Muray devait penser en parallèle la persistance dans l'être du Moi littéraire et la mutation anthropologique incubée par l'entrée dans l'ère post-historique. Enfin, Barrès et Cioran se rejoignent dans une même oscillation entre la fascination pour diverses formes de radicalisme ou d'extrémisme politique d'une part, et des préoccupations exclusivement littéraires d'autre part. Cependant, la trajectoire de Cioran peut être résumée à grands traits par un passage de la première attitude à la seconde, quand celle de Barrès apparaît plus fluctuante, les aventures politiques et littéraires se succédant parfois, s'entremêlant souvent.

Sur ce point, Barrès et Cioran semblent se rapprocher l'un de l'autre au détriment de Muray: ce dernier n'a en effet jamais participé activement à un mouvement politique déterminé, se contentant d'appartenir, de par sa date de naissance - 1945 - bien plus qu'en vertu d'un corpus idéologique partagé, à une génération intellectuelle. Cioran au contraire, bien qu'il n'appartînt jamais à un quelconque parti, ne fut pas insensible au projet de révolution fasciste porté par la Garde de Fer, comme en témoigne l'opuscule de 1936 La Transfiguration de la Roumanie. Cet engagement de jeunesse, conséquence pratique du désir de se joindre à une action collective dont les excès mêmes ne pouvaient que séduire un Moi exacerbé, ne fut pas pour rien dans le choix ultérieur du scepticisme radical et de la tentation d'abandon du Moi.

Ce point de contact du Moi et de l'Histoire conduisit enfin Maurice Barrès vers des choix politiques toujours embarrassants pour ses admirateurs. Mais ce qui importe pour notre réflexion n'est pas que ses engagements aient pu prendre la forme d'une farce rétrospective avec le boulangisme et la tentative de coup d'État de Déroulède, ni que l'emphase excessive de certaines chroniques de guerre prête au mieux à sourire, ni même que l'on ne puisse lire le récit de la dégradation de Dreyfus sans se frotter les yeux. L'enjeu ici est de dresser le constat que Barrès, voulant mener à son terme un projet formalisé en 1888-1891 - prendre part à une aventure historique, armé d'un Moi préalablement sublimé par le dandysme individualiste -, ne parvint qu'à compromettre sa propre postérité par les moyens mêmes qui devaient l'assurer. À l'inverse, Cioran tira de ses élans de jeunesse la volonté d'une critique radicale, adressée conjointement au Moi et à l'Histoire, l'un et l'autre ne pouvant exister ou s'annihiler que de concert, comme le postule la lettre à Constantin Stoïca:

Celui qui, avant la trentaine, n'a pas subi la fascination de toutes les formes d'extrémisme, je ne sais si je dois l'admirer ou le mépriser, le considérer comme un saint ou un cadavre. Faute de ressources biologiques, ne s'est-il pas placé au-dessus ou au-dessus du temps? (Cioran, 2007: 980-981) 
Le recul critique, comme la totalité du geste littéraire cioranien, ne peut aller sans une forme d'humour qui ne s'épanouit jamais mieux qu'en prenant son propre Moi comme objet de sarcasme. Dans la même lettre, le penchant démocratique apparaît ainsi explicitement comme l'unité de mesure de la déchéance du Moi. Mais cette dernière se voit paradoxalement sublimée par une intransigeance constituant le dernier souvenir des excès de jeunesse du penseur, retournés pour ainsi dire contre eux-mêmes:

$\mathrm{Au}$ fur et à mesure que mon énergie déclinait, s'accentuait mon penchant à la tolérance. Décidément, je n'étais plus jeune: l'autre m'apparaissait concevable et même réel. Je faisais mes adieux à l'Unique et sa propriété; la sagesse me tentait: étais-je fini? Il faut l'être pour devenir un démocrate sincère. À mon grand bonheur, je m'aperçus que tel n'était pas exactement mon cas, que je conservais des traces de fanatisme, quelques vestiges de jeunesse: je ne transigeais sur aucun de mes nouveaux principes, j'étais un libéral intraitable (982-983).

Le parcours politique de Philippe Muray n'a quant à lui jamais croisé la route d'aucune forme d'extrémisme, ce qui semble à première vue le mettre à part de Barrès et Cioran. Toutefois, l'un de ses sujets d'étude privilégiés fut Céline; plus précisément, la non-séparabilité du romancier de génie et du pamphlétaire antisémite est l'une des thèses centrales de l'essai de 1981:

Quelle passion nous pousse à vouloir qu'il y ait deux Céline, un Céline impeccable, hygiénique, marionnette lustrée ressortie pour les parades euphoriques de l'avant-garde, et un Céline sordide, contaminé, définitivement enterré dans les cloaques de l'Histoire? Quelle cause commune, quel sens commun, quel intérêt férocement collectif avonsnous à ce que Céline soit coupé en deux? Enfin, à quel conformisme voulons-nous faire servir cette moitié de Céline, ce demi-Céline, ce Céline au détail, bissectionné? Et à quelle part de nuit et de cauchemar en nous-mêmes avons-nous décidé de jeter en pâture secrète la parcelle non lotie de son œuvre ainsi équitablement partagée entre tous les héritiers? (Muray, 2001: 47)

Cette série de questions trouve en réalité une réponse globale dans les toutes premières phrases de la "Préface à la nouvelle édition":

Le nom de Céline appartient à la littérature, c'est-à-dire à l'histoire de la liberté. Parvenir à l'en expulser afin de le confondre tout entier avec l'histoire de l'antisémitisme, et ne plus le rendre inoubliable que par-là, est le travail particulier de notre époque, tant il est vrai que celle-ci, désormais, veut ignorer que l'Histoire était cette somme d'erreurs considérables qui s'appelle la vie, et se berce de l'illusion qu'on peut supprimer l'erreur sans supprimer la vie (9).

L'essai n'a donc pas pour unique objet de réduire à néant la bonne conscience d'un lecteur qui prétendrait admirer le style célinien en occultant l'antisémitisme qui en est indissociable: il ouvre également la porte à l'une des idées centrales de Muray, l'entrée dans 
l'ère post-historique, le refoulement collectif de l'Histoire. Un entretien de 2002 avec Olivier Rohe résume parfaitement cette vision:

L'Histoire à mes yeux n'aura été, au fil des siècles, que l'ensemble des forces qui, consciemment ou pas, le plus souvent inconsciemment, et souvent aussi dans la plus grande confusion, mais toujours avec une fermeté extrême, ont tenté de ne pas arriver à l'état dans lequel nous nous trouvons aujourd'hui (Muray, 2010: 1217).

De manière évidente, selon Muray, la reconnaissance de la non-séparabilité du meilleur et du pire au sein d'un même Moi est l'un des signes distinctifs de l'ère historique. Par conséquent, aux yeux de l'auteur, l'œuvre est l'unique modalité viable d'insertion du Moi littéraire dans l'Histoire, puisque la littérature est en elle-même une condition d'existence de la continuité historique. À l'inverse, lorsqu'un écrivain s'engage dans un mouvement de masse - en s'exposant au choix des pires causes possibles puisque les paradigmes du Moi littéraire menacent de court-circuiter le discernement du Moi social -, sa personne et son œuvre ne peuvent qu'être expulsées ou déformées par la mémoire collective, une fois entérinée l'entrée dans l'ère post-historique. Difficile par ailleurs, de ne pas penser ici à la postérité pour le moins mutilée de Barrès. Il semblerait donc que les trois points de Céline puissent constituer une ligne en pointillés qui lierait, par-delà Cioran, Barrès et Muray ${ }^{8}$.

Ce dernier précisément est, des trois auteurs, celui qui a le plus constamment orienté sa réflexion vers l'étude du Moi littéraire, mais également celui aux yeux duquel la figure de l'écrivain engagé - autrement dit le Moi social prétendant prendre part à l'Histoire en mobilisant le Moi littéraire - apparaissait comme une absurdité incarnée. Cet extrait des Exorcismes spirituels II établit un lien causal entre le déclin de la littérature et l'extension de sa frange "militante":

Le $\mathrm{XX}^{\mathrm{e}}$ siècle n'aura apprécié, en fin de compte, que les œuvres où les idées militent. Où les images militent. Où les formes, les couleurs, les paroles militent. La morale est le pôle Nord de l'interprétation littéraire contemporaine. Sans morale, plus de boussole. Plus de procès, plus de ressentiment, plus de reproches possibles. Plus de communauté (Muray, 2010: 850).

Un positionnement à la fois explicité et nuancé dans le texte "Du confort intellectuel aux intellectuels de confort" dans les Exorcismes spirituels III: Muray y postule la nécessité d'une "critique artistique" du monde contemporain, en opposant, à la figure de l'intellectuel français dont l'engagement affiché résume le geste littéraire, celle de l'artiste (Voltaire,

8 Une hypothèse que nous nous devons de nuancer en notant l'absence apparente de Barrès dans le panthéon littéraire murayien: l'on ne relève qu'une occurrence de son nom tout au long des Exorcismes spirituels, au début de l'article "Crépuscule des lieux". Muray y réfute l'ouverture de la Colline inspirée ("il est des lieux où souffle l'Esprit”), en lui opposant l'idée que les lieux n'acquièrent un “esprit» qu'une fois sublimés par la vision d'un peintre. Voir Muray, 2010: 1031. 
Diderot, Hugo, Zola, Sartre) tirant de son œuvre la légitimité indispensable à un éventuel engagement ultérieur (1302-1303). L'essayiste applique d'ailleurs la même grille de lecture à son propre travail, en définissant sa critique des mœurs contemporaines comme une prise de position esthétique plutôt que socio-politique:

\begin{abstract}
Mon esthétique essaie de coller le plus étroitement possible au sujet que je traite. Ce sujet étant de l'ordre de l'énormité systématique, puisqu'il s'agit de notre époque, il est nécessaire de démesurer la forme et le fond pour l'exprimer (1329).

Mes préoccupations sont esthétiques, non doctrinales. La critique des conditions d'existence actuelles que j'élabore est inséparable d'une prise de position d'ordre artistique. C'est parce que mon jugement est esthétique (non philosophique ou sociologique) que je peux apprécier comme elles le méritent toutes les prétendues innovations de notre temps $[\ldots](1347)$.
\end{abstract}

Là encore, une continuité semble exister, par-delà Cioran, entre le romancier et l'essayiste. Car le parcours barrésien illustre, à lui seul, plusieurs des théories murayiennes quant à l'inscription active de l'écrivain dans l'Histoire: le positionnement intellectuel justifié et renforcé par les paradigmes développés dans l'œuvre, l'arrière-plan esthétique surdéterminant les enjeux de l'engagement, mais aussi la difficulté à faire coïncider l'individualisation à outrance du Moi littéraire avec la lucidité politique du Moi social. Que Maurice Barrès n'ait pas toujours brillé par sa pertinence dans le choix des causes à défendre ne doit donc pas nous faire oublier la cohérence sous-jacente de son parcours, qui consista parallèlement à élever son Moi à la hauteur du temps historique et à rechercher dans l'histoire contemporaine un champ de bataille où exercer son Moi. Sarah Vajda abonde dans ce sens en rappelant que l'activité de chroniqueur de guerre représente l'aboutissement logique de toute l'œuvre barrésienne:

Oublier la Chronique signifierait oublier les pages où Barrès a atteint un but poursuivi toute sa vie, l'annihilation de son Moi au profit d'un Nous, geste par lequel l'écrivain né au siècle du 'sacre de l'écrivain' tendait la main à Chrétien de Troyes, à Joinville, aux mémorialistes du temps jadis, geste admirable par lequel un auteur consacré remettait en jeu la mise acquise et l'offrait à tous, se séparant pour jamais des cénacles et de l'élite. (Vajda, 2000: 27).

Ici, le chroniqueur rejoint effectivement le romancier, dans la mesure où "l'annihilation du Moi au profit d'un Nous" est précisément, dans La Colline inspirée, le stade que Léopold Baillard ne parvient jamais à atteindre. Le dévouement désiré se heurte en effet perpétuellement à un Moi qui parodie son rôle historique à défaut de pouvoir authentiquement le remplir. Le discours qu'il prononce lors de la procession, censé marquer son triomphe et son apogée, s'embourbe ainsi dans un verbiage qui ne parvient qu'à révéler à l'assistance l'infatuation stérile d'un Moi boursouflé: 
Mais Léopold avait trop de hâte d'épancher son cœur. Il oubliait d'établir les faits les plus essentiels, de donner un historique de son voyage; il supposait connus Vintras et Tilly. Dans le prodigieux effort qu'il faisait pour traduire les sentiments qui venaient depuis trois mois d'émerger de son âme profonde, et pour tenter avec eux une éducation nouvelle de ses paroissiens, il ne pensait plus à raconter son aventure, mais simplement à exprimer le frisson lyrique dont elle l'avait remué. Ses paroles pleines de son et de cadence, plus qu'à leur ordinaire, car la passion la plus vraie l'enfiévrait, mais trop obscures, passaient par-dessus la tête des auditeurs, et s'envolant du plateau, au-delà des buissons des pentes, allaient au loin retomber comme des semences invisibles (Barrès, 1996: 135).

Plus loin, la tentation pour le personnage de voir dans son action la preuve de sa nature christique, aggrave ce constat:

Ces sensations miraculeuses et l'approche du danger eurent pour effet d'exalter chez Léopold le sentiment de la personnalité. Son esprit échauffé fit une construction singulière: il se persuada que la semaine sainte qui s'ouvrait allait reproduire pour lui, sur cette montagne, au milieu de paysans ingrats, tout ce que le Christ avait souffert, en Judée, d'une foule ameutée par les princes des prêtres et des pharisiens. Imagination qui n'a rien pour surprendre, chez un homme dont le rêve fut toujours de calquer sa vie sur des patrons sublimes. Et pour commencer, ce dimanche allait être vraiment son dimanche des Rameaux: entouré de ses fidèles - à l'occasion de son déménagement - il allait faire son entrée dans Jérusalem (Barrès, 1996: 224).

Ainsi, revenant, après son exil, près de son ancien couvent désormais délaissé, la vue des ruines incite moins Léopold à méditer sur son échec qu'à se voir lui-même comme un "reproche incarné" adressé à ses anciens ennemis:

Mais cette solitude, bien faite pour affliger son cœur, eut cet effet inattendu de surexciter son orgueil. Ces ruines désespérées affirmaient la grandeur de ses conceptions et l'injustice de son exil; elles parlaient pour lui. Les années avaient passé sans qu'il fût remplacé. Chacune de ces pierres, en tombant, jetait un amer reproche à l'évêque de Nancy: 'Vous nous avez prises à celui qui nous aimait, et vous ne savez rien faire de nous. Monseigneur, comme tout cela vous accuse!' (276).

Le dialogue final entre l'Église (allégorie de l'ordre et de la collectivité) et la Prairie (symbolisant l'enthousiasme individuel), s'il conclut le roman sur l'interdépendance entre les deux entités (donc en définitive, entre le Nous et le Moi), s'achève toutefois sur une reconnaissance de la primauté de la première sur la seconde: “L’Église est née de la prairie, et s'en nourrit perpétuellement - pour nous en sauver» (393). L'insertion finale du "pour nous en sauver" donne ainsi, au propre comme au figuré, le dernier mot du roman au Nous.

La vision cioranienne de l'Histoire entre donc en dissonance, tant avec l'acte de décès établi par Muray qu'avec la tentative de renaissance rêvée par Barrès. En effet, si ces deux derniers ont reconnu dans l'Histoire une réalité substantielle face à laquelle le Moi doit se 
positionner, le penseur roumain lui a donné le même statut d'illusion indépassable qu'au Moi et au Nous. "Mécanismes de l'utopie", dans Histoire et Utopie, illustre cette approche essentiellement négative du mouvement historique, en postulant que tout changement de société doit plus au désir de renverser un ordre établi que de lui en substituer un nouveau: “Lorsqu'on est excédé des valeurs traditionnelles, on s'oriente naturellement vers l'idéologie qui les nie. Et c'est par sa force de négation qu'elle séduit, bien plus que par ses formules positives" (Cioran, 2007: 1045). Le lien entre la nature du Moi et la possibilité de l'inscrire dans l'Histoire est quant à lui évoqué dans “À l'école des tyrans":

A-t-on remarqué la symétrie? Pour devenir homme politique, c'est-à-dire pour avoir l'étoffe d'un tyran, un dérangement mental est nécessaire; pour cesser de l'être, un autre dérangement s'impose non moins: ne s'agirait-il pas, au fond, d'une métamorphose de notre délire des grandeurs? Passer de la volonté d'être le premier dans la cité à celle d'y être le dernier, c'est, par une mutation de l'orgueil, substituer à une folie dynamique une folie statique, un genre insolite d'insanité, aussi insolite que le renoncement qui en procède, et qui, relevant de l'ascèse plutôt que de la politique, n'entre pas dans notre propos (1006-1007).

Le "dérangement mental" nécessaire tout autant à l'affirmation démesurée du Moi dans le temps historique ("folie dynamique") qu'au désir de s'en retirer ("folie statique") ressemble à s'y méprendre au "déséquilibre vital" qui, dans les Cimes du Désespoir, conditionnait et précédait nécessairement l'entrée dans la pensée spéculative (32): une même affectation du Moi semble donc être, aux yeux du Cioran, à l'origine de la création littéraire tout autant que de la dynamique historique. En parallèle, dans "L'âge d'or", le penseur renverse cette proposition dans une perspective plus proche de celle de Muray. Prenant l'exemple de Dostoïevski se "vengeant" sur les héros des Démons des utopies de sa jeunesse, Cioran postule qu'un engagement reconnu comme une erreur constitue une source d'inspiration littéraire de premier plan (1057-1058).

Toutefois, lorsque Cioran évoque son propre parcours, ce n'est pas la nature de l'engagement, mais la volonté même d'un engagement dans la dynamique historique, qui est représentée sous les traits d'une erreur de jeunesse. Car le Moi et l'Histoire ont, dans la perspective cioranienne, en commun d'être des entités à la fois illusoires par leur nature objective, et incontournables par le rôle qu'elles jouent nécessairement dans l'élaboration de toute pensée. Et là encore, la raison de cette volonté contrariée d'annulation de l'Histoire était donnée dès les Cimes du Désespoir: "L'éternité ne mène ni au triomphe du bien ni à celui du mal: elle annule tout" (61). L'antagonisme entre les limites temporelles de l'expérience humaine et la pensée de l'éternité conditionne par définition le sens - ou l'absence de sens - donné par Cioran à la confrontation entre le Moi et l'Histoire:

L'éternité ne se laisse comprendre qu'en tant qu'expérience, comme quelque chose de vécu. La concevoir objectivement n'a aucun sens pour l'individu, car sa finitude tem- 
porelle lui interdit d'envisager une durée infinie, un processus illimité. L'expérience de l'éternité dépend de l'intensité des réactions subjectives, l'entrée dans l'éternité ne peut s'accomplir qu'en transcendant la temporalité (62).

Le chapitre "Histoire et éternité"conclut logiquement ces développements en affirmant que l'éternité annule nécessairement la temporalité dans son ensemble (64). Le raisonnement est quasi-mathématique: toute temporalité est nulle au regard de l'éternité de même que tout nombre équivaut à zéro au regard de l'infini.

Ici, c'est le Spinoza des Principes de la Philosophie de Descartes qui peut nous donner le fin mot de la vision cioranienne: “Ainsi le temps n'est pas une affection des choses, mais seulement un simple mode de pensée, ou, comme nous l'avons déjà dit, un être de raison; c'est un mode de pensée qui sert à l'explication de la durée" (Spinoza, 2002: 258). Ainsi, reconnaissant le temps comme une expérience propre au Moi, et par conséquent l'Histoire comme la traduction, dans un langage collectif, de cette expérience, Spinoza nous éclaire sur l'interdépendance entre l'écriture du Moi et la pensée de l'Histoire propre à chacun des trois auteurs. Maurice Barrès a mis en pratique une théorie selon laquelle le devoir du Moi littéraire serait d'atteindre son plus haut point d'individuation dans le but de contribuer à sublimer le destin historique du Nous national; ce faisant, son Moi social entraîna pendant un temps son propre Moi littéraire dans l'antidreyfusisme acharné. Si bien que ces lignes de Philippe Muray s'appliquent parfaitement à l'auteur du Culte du Moi:

Il faut donc s'être mis dans son tort, d'une façon ou d'une autre, pour réenchanter le jeu, jouir, mettre en échec la cohésion des apparences qui ont raison, faire de la grande littérature en somme? Pas nécessairement. Mais tout de même, tout de même... Voyez Chateaubriand, Dostoïevski, Dante, Balzac, Céline et bien d'autres. Épousant des causes perdues d'avance. Géniaux losers acharnés. Très loin de la cohorte des victorieux sourds et bien portants. Cherchant l'électrochoc de la perte d'innocence. C'est l'innocence qui tremble de trouille dans son auto-glorification, jamais la culpabilité qui se connaît (Muray, 2010: 926).

Philippe Muray, justement, n'a cessé de penser en parallèle la fin de l'Histoire et le Moi littéraire des auteurs auxquels son œuvre critique s'est consacrée: le second apparaît dans ses écrits, à la fois comme un signe distinctif du temps historique et comme une survivance à même de mettre en scène l'ère post-historique en tant qu'objet littéraire. "En attendant que nos maîtres nous aient refait des certitudes, il convient que nous nous en tenions à la seule réalité, au moi.", écrivait Barrès en 1888 (Barrès, 1986: 18). Muray aurait pu reprendre à son compte la seconde partie de la phrase; la première partie en revanche n'aurait eu aucun sens sous la plume, puisque l'auteur d'Après l'Histoire pensait, justement, que le temps des "maîtres" et des "certitudes à refaire" appartenait à un passé révolu. Cioran, quant à lui, a bâti une œuvre dans laquelle le Moi, le Nous et l'Histoire partageaient en théorie la même inconséquence et la même absurdité. Mais dans les faits, la quête et l'effort de définition du 
Moi demeura le plus petit dénominateur commun à tous ses écrits, et l'Histoire, quoique théoriquement annulée par la conscience de l'éternité, ne cessa jamais de compter parmi les thématiques structurantes de sa pensée.

Définir le Moi littéraire comme un apophatisme suppose, en premier lieu, de tourner ses regards vers des auteurs au Moi suffisamment hypertrophié pour être saisissable comme objet de pensée, et assez pétri de contradictions pour que sa nature indéfinissable apparaisse en pleine lumière. De ce point de vue, la confrontation entre l'évolution littéraire de Maurice Barrès et ses errements politiques, l'œuvre aphoristique et perpétuellement en tension d'Emil Cioran, et l'interpénétration de l'exégèse littéraire ${ }^{9}$ et de la critique de mœurs chez Philippe Muray, nous a semblé fournir un sujet d'étude idéal. Le Moi littéraire peut relever de la théologie apophatique en ce qu'il représente une entité à la fois inconnaissable et incontestable; les opposés qui le définissent négativement peuvent être groupés sous la dénomination du Nous, contrepoids indispensable à son émergence et à son affirmation. Par ailleurs, la perception par l'écrivain de son inscription au sein de la communauté surdétermine très largement sa vision de l'histoire humaine et sa volonté de s'y insérer.

Ainsi, Maurice Barrès est, des trois auteurs, celui qui a le plus nettement explicité la nature aphairétique de la quête du Moi, par le titre donné à la trilogie de 1888-1891 et les différentes étapes de l'évolution de Philippe. Plus encore, son propre parcours, allant du dandysme égocentré au "professeur d'énergie", révèle que la séparation du Moi et du Nous n'était pas à ses yeux un absolu, mais une étape nécessaire à la définition du Moi, elle-même préalable à son inscription dans le cours de l'Histoire. Le cas de Cioran répond à celui de Barrès, en ce que la volonté de prendre part à un tournant historique représente la première étape de son évolution intellectuelle. Le recul critique pris par le penseur sur ses élans de jeunesse fut finalement englobé par une remise en cause radicale - quoique théorique - de la réalité du Moi. Pour autant, l'existence du Moi et la conscience historique ne furent jamais niées en tant que sources et conditions de sa pensée. Le "culte apophatique" du Moi par Cioran apparait donc sous une forme dialectique plutôt qu'essentialisée. Philippe Muray au contraire, a élaboré une grille de lecture dans laquelle la nature négative du Moi de l'écrivain constitue une condition d'existence de son œuvre. Par ailleurs, ayant perçu dans le tournant des $\mathrm{XX}^{\mathrm{e}}$ et $\mathrm{XXI}^{\mathrm{e}}$ siècles une période de sortie de l'Histoire, il fit du Moi littéraire l'unique possibilité de conserver la mémoire du temps historique, puisque ce dernier, dans sa pensée, n'était plus pensable qu'en tant qu'objet littéraire.

9 Et picturale: quoique cet aspect du parcours de Muray n'ait pas été au cœur de notre réflexion, mentionnons toutefois ses peintures de jeunesse, et rappelons que les Exorcismes spirituels multiplient les études sur de nombreux peintres (Cézanne, Delacroix, Soutine, Degas, etc.). Muray est par ailleurs l'auteur de l'essai La Gloire de Rubens (Grasset, 1991, réédité en 2013 par Les Belles Lettres). 
Du Moi comme apophatisme: Maurice Barrès, Emil Cioran, Philippe Muray

\section{Références bibliographiques}

BARRÈs, Maurice. 1986. Le Culte du moi. Sous l'œil des barbares, Un homme libre, Le jardin de Bérénice. Paris, 10/18, coll. "Fins de siècles".

BArrès, Maurice. 1996. La Colline inspirée. Paris / Genève, Slatkine, coll. "Fleuron".

Cioran, Emil. 2007. "Sur les Cimes du Désespoir", "Syllogismes de l'Amertume", "La Tentation d'exister", "Histoire et Utopie", "La Chute dans le Temps", "De l'Inconvénient d'être né” in Euvres. Paris, Gallimard, coll. "Quarto", 15-102, 743-813, 819-970, 977-1061, 1069-1158, 1269-1400.

Muray, Philippe. 2001. Céline. Paris, Gallimard, coll. "Tel”.

Muray, Philippe. 2010. "Exorcismes spirituels. I, II, III, IV" in Essais. Paris, Les Belles Lettres, 457-770, 771-1121, 1123-1451 , 1453-1764.

Muray, Philippe. 2013. La Gloire de Rubens. Paris, Les Belles Lettres.

Pascal, Blaise. 2011. Pensées, opuscules et lettres. Paris, Classiques Garnier (coll. Bibliothèque du XVII ${ }^{\mathrm{e}}$ siècle).

Plotin, 1861. Ennéades. Tome troisième. Paris, Librairie Hachette.

Proulx, François. 2011. "De nouveaux et étranges éducateurs: dangers de la lecture et remèdes littéraires, 1883-1914" in Culture \& Musées, n¹7, 21-40.

Spinoza, 2002. Euvres complètes. Paris, Gallimard, coll. "Bibliothèque de la Pléiade".

VAJdA, Sarah. 2000. Maurice Barrès. Paris, Flammarion, coll. “Grandes Biographies”. 